\title{
Multimedia communications in vehicular adhoc networks for several applications in the smart cities
}

\author{
Cristhian Iza Paredes, José Antonio Uribe Ramírez, Nely P. López Márquez, Leticia Lemus, Ahmad \\ M. Mezher, Mónica Aguilar Igartua \\ Departamento de Ingeniería Telemática, \\ Universidad Politécnica de Catalunya (UPC) \\ Campus Nord UPC. Despacho C3-301. C/ Jordi Girona 1-3. 08034 Barcelona.
}

\{cristhian.iza, antonio.uribe, leticia.lemux, nely.lopez, ahmad.mezher, monica.aguilar\}@entel.upc.edu

\begin{abstract}
Resumen- Road safety applications envisaged for vehicular ad hoc networks (VANETs) depend largely on the exchange of messages to deliver information to concerned vehicles. Safety applications as well as inherent VANET characteristics make data dissemination an essential service and a challenging task. We are developing a decentralized efficient solution for broadcast data dissemination through two game-theoretical mechanisms. Besides, VANETs can also include autonomous vehicles (AVs). AVs might represent a revolutionary new paradigm that can be a reality in our cities in the next few years. AVs do not need a driver to work; instead, they should copy a proper human behavior to adapt the driving according to the current circumstances, such as speed limit, pedestrian crossing street or wheather conditions. We will develop an AV software module including artificial intelligence (AI) techniques so that AVs can interact with the dynamic scenario throughout time. Finally, we also will include electrical vehicles (EV) in the VANET, so that special services such as finding and reserving an EV charging station place will be welcome. In addition, we are developing a multimetric geographic routing protocol for VANETs to transmit $\mathbf{H . 2 6 5}$ video (traffic accident, traffic state, commercial....) over VANETs.
\end{abstract}

Palabras Clave- Vehicular Ad hoc NETworks, video dissemination, privacy in VANETs, electrical vehicle $(\mathrm{EV})$, autonomous vehicle (AV), artificial intelligence (AI).

\section{INTRODUCCIÓN}

Vehicular ad hoc networks (VANETs) are foreseen as an essential component of the future intelligent transportation systems (ITS) to support safety, traffic management, and user infotainment applications. VANETs are a type of mobile ad-hoc networks (MANETs) forming self-organized networks without the requirement of permanent infrastructure, in which nodes are vehicles. Thus, the network topology might change rapidly due to the high mobility of nodes (vehicles), which makes communications a challenge.

VANET vehicles can stablish vehicle-to-vehicle (V2V) communications to share information, as well as vehicle-to-infrastructure (V2I) communications for other kind of services. VANETs are intented for safeapplications, traffic management, and enhanced navigation among other services.

Today, road traffic is the ninth biggest cause of death worldwide. By 2030, the increase in the number of vehicles will see road traffic become the fifth largest cause of death. One of the most important uses of VANETs are safety applications, which usually rely on broadcast-based algorithms. Flooding-based algorithms can be used to disseminate emergency messages rapidly and efficiently through the VANET to warn vehicles around $e . g$. an accident. Thus, a key research problem is the design of a scalable dissemination scheme, which is efficient, reliable and incurres short delay under different VANET conditions. To tackle this issue, we have developed an Adaptive Distributed Dissemination (ADD) [1] protocol, based on game-theoretical algorithms to compute the forwarding probability of nodes based on several design parameters. 
In the considered VANETs, we can find autonomous vehicles (AVs) and electrical vehicles $(\mathrm{EV})$, which have special features and necessities that we will consider in our simulated scenarios. For instance, AVs must communicate not only with other vehicles through the VANET, but also with the infrastructure in the city (e.g. traffic lights), with pedestrian crossing the streets and with any possible obstacle around. In addition, AVs must impersonate the human behaviour to take proper driving decisions (e.g. brake, accelerate, stop...). To do so, we will include artificial intelligence (AI) algorithms in the AV module. On the other hand, EVs have special needs. For instance they require electric vehicle charging stations (EVCS) to recharge their batteries during their trip if necessary. Thus, it would be basic to develop a smart service so that EVs could reserve an electrical recharging point in special EVCS deployed in the city.

Another goal of our research team is the development of a multimetric geographical routing protocol for VANETs to transmit H.265 video. Possible applications include: (a) the transmission of a short video clip about an accident to alert quickly the smart emergencies services (e.g. 112 or 911) in the city; (b) a light video to report the state of the traffic to the traffic management unit in the city; (c) the transmission of a short light video advertisement for the passengers about restaurants or shops discounts around, among other examples. Furthermore, sometimes, it is not necessary to send the exact geographic location (e.g. GPS location) of the vehicle in order to keep the privacy of the user (e.g. not to be tracked). To do so, we will apply microagregation techniques [8].

\section{AN ADAPTIVE GAME-THEORETICAL DISTRIBUTED DISSEMINATION PROTOCOL FOR VANETS}

Road safety applications for VANETs rely on the dissemination of warning messages to deliver information to vehicles near e.g. an accident. We have proposed an Adaptive Distributed Dissemination (ADD) protocol [1] to perform data dissemination in VANETs. ADD is designed to operate without any roadside infrastructure in urban scenarios under diverse road traffic conditions. ADD uses a decentralized solution for the broadcast data dissemination problem through two game-theoretical mechanisms. Using game theory (GT), we have designed a mechanism to predict behavior in situations where a state is the result of a series of interactions between different nodes (players), who act according to their preferences regarding future performance and existing incentives. In first place, the Asymmetric Volunteers Dilemma Game is evaluated as a mechanism to tackle the broadcast storm problem. The probability that a node forwards a broadcast message is calculated using the number of candidate vehicles to forward the message, i.e., the number of vehicles that are listening to the transmission. The cost/benefit relation to forward the message by a vehicle is obtained from metrics like distance (to an intersection, from the incident, to an access point...), average packet delay and link quality. Also, the Forwarding Game is designed as a second mechanism to mitigate the broadcast storm problem. In this case, the strategy of the players consists on selecting a forwarding probability that maximizes the pay-off using a utility function, which depends on the player's availability and the forwarding probability of other players. Availability of a player is a normalized factor based on several metrics. Our proposal ADD considers the position of the vehicle in the network (distance between receiver to next junction, distance between transmitter and receiver), an estimation of the link quality (derived from signal quality, channel quality and collision probability), time elapsed since the generation of the message and estimated available bandwidth. Nodes themselves calculate those metrics and broadcast them in periodic beacons to their neighbours.

Extensive simulations reveal that our proposal excels in terms of number of transmissions, lower endto-end delay and reduced network load while maintaining high delivery ratio, compared to other proposals.

\section{Multimetric QOS-AWARE GEOGRAPHIC ROUTING PROTOCOL FOR VANETS}

We start from the well-known GPSR (Greedy Perimeter Stateless Routing) [7], which uses the position of the nodes to take packet forwarding decisions. GPSR uses greedy forwarding to forward packets to nodes that are always progressively closer to destination. In regions of the network where such a greedy path does not exist (i.e., the only path requires that one move temporarily farther away from the destination), GPSR recovers by forwarding in perimeter mode, in which a packet traverses successively closer faces of a planar subgraph of the full radio network connectivity graph, until reaching a node closer to destination.

Our multimetric geographical routing protocol for VANETs, takes into account several metrics to take the best hop-by-hop forwarding decisions under the current circumstances. We consider distance to destination, vehicle's velocity, vehicles' trajectory, vehicles' density, available bandwidth and packet losses. The design of the metrics is detailed in [9] and here we will just summarize one of them, the vehicles' density.

Vehicles' density: It is computed as the number of vehicles $\left(\mathrm{N}_{\mathrm{v}}\right)$ in the neighbors' list of each node at the moment of sending the current hello message, divided by the area within the transmission range $\left(\pi . T R^{2}\right)$ of that vehicle, being $T R$ the transmission range. Each node computes its density of nodes $\rho_{N g h}$ using Eq. (1) and includes it in the next hello message.

$$
\begin{aligned}
& \rho_{N g h}=\frac{\mathrm{N}_{\mathrm{V}}}{\pi \cdot T R^{2}} \\
& u_{d n s, N g h}= \begin{cases}\frac{-1}{\rho_{\max }{ }^{2}} \rho_{N g h}{ }^{2}+\frac{2}{\rho_{\max }} \rho_{N g h} & \text { if } \rho_{N g h} \leq 2 \rho_{\max } \\
0, & \text { if } \rho_{N g h}>2 \rho_{\max }\end{cases}
\end{aligned}
$$


Neighbors' lists are updated upon reception of new hello messages, which are sent by nodes (usually one per second) within their neighbourhood. The algorithm gives a higher score when the neighbor node $N g h$ has a higher value of $\rho_{N g h}$. Nodes with a denser area in the transmission range will have more possibilities to forward the packet to a next node. This is true until a maximum nodes' density $\rho_{\max }$, above which there are too many vehicles and the collisions' probability increases. We set $\rho_{\text {max }}$ to 200 vehicles $/ \mathrm{km}^{2}$.

We have designed a concave function for the density metric shown in Fig. 1. This function has its maximum at $\rho_{N g h}=\rho_{\max }$ and above $\rho_{\max }$ it decreases till $2 \rho_{\max }$ where again it reaches zero and keeps on zero for $\rho_{N \text { gh }}>2 \rho_{\text {max }}$.

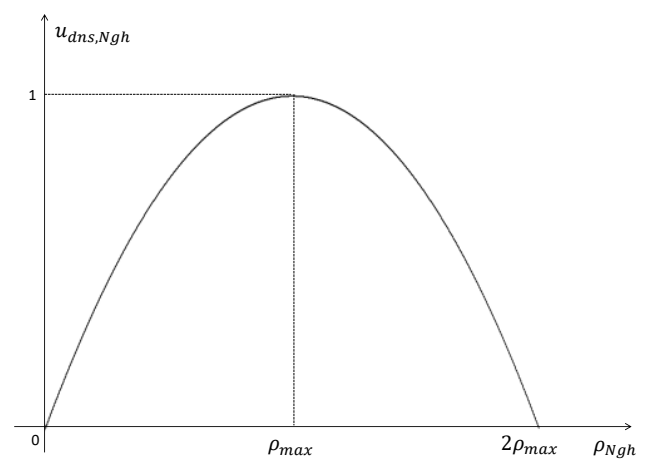

Fig. 1. Designed metric for the vehicles' density, $u_{d s t, N g h}$.

Eq. (2) describes how we calculate the vehicles' density metric $u_{d n s, N g h}\left(0 \leq u_{d n s, N g h} \leq 1\right)$ as shown in Fig. 8 . This way, we penalize those nodes whose number of neighbors in their transmission range is above a threshold $\left(\rho_{\text {Ngh }}>\rho_{\max }\right)$.

Furthermore, we will include machine learning (ML) techniques to take best forwarding decisions at each moment by weighting all considered metrics with proper dynamic weights. The idea is to test machine learning models (e.g., trees, logistic regression, $\mathrm{KNN}$ ) to see which one predicts better (higher accuracy). This way, the performance evaluation of our proposal will notably improve the quality of service (QoS) offered by other similar routing protocols.

\section{MACHINE LEARNING AND PRIVACY IN VANETS}

Machine learning (ML) is a scientific discipline in the field of artificial intelligence (AI) that uses systems that learn automatically. Learning in this context means identifying complex patterns in tones of data. The machine that learns is an algorithm that checks data and is able to predict future behavior. ML systems improve autonomously over time without human intervention

The use of machine learning in VANETs is an idea to improve the performance of this kind of networks. Specifically, we plan to use principal component analysis (PCA), which is a technique used to emphasize variations and extract patterns from a dataset. The first step is to apply a ML technique offline. Then, use PCA online to make a self-learning to choose the best routes to transfer the information, trying to decrease the packet losses in the network. PCA will provide in an offline analysis the distribution of energies that each metric represents. Thus, we could estimate the correct weights of each one of the considered metrics. After that, and by recalculating the correct weights instead of giving equal weights, best forwarding routes will be chosen. To improve the results, we will test different ML algorithms to find the one that performs better in VANETs.

On the other hand, privacy is a right to be free from secret surveillance and to determine whether, when, how, and to whom, one's personal information is to be revealed. ML techniques allow us to hide important information, although keeping the proper functioning of the network. We claim that vehicles have the right to choose what information want to share or want to make public, so in this scenario ML is a good tool that can help VANETs to keep information secure.

\section{REALISTIC SIMULATION PLATFORM}

In order to attain realistic simulations to trust the obtained results from our proposals, it has paramount importance to prepare a realistic simulation platform. In our case, we use OMNeT++ [2] to perform the simulations and SUMO [3] to generate the vehicular movement traces. OMNeT++ provides a baseline to develop different type of projects which implement the actual simulators. Two of these projects can be used together to provide a vehicular network simulator, INET [4] and VEINS [5]. For a more realistic mobility behavior, we defined a scenario including different type of vehicles (car, bus and truck) with an associated probability of occurrence. Besides, seeking to dispose a scenario prepared as much realistic as possible, we use real maps extracted from OpenStreetMap [6]. This simulation platform allows us to include realistic features of real world maps and to interact with realistic vehicular mobility models.

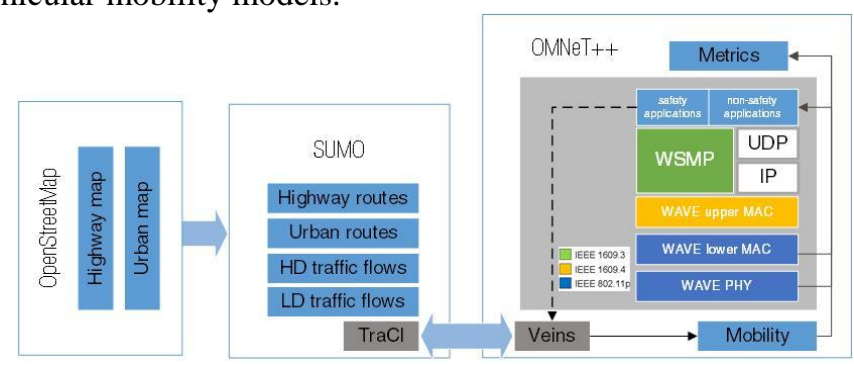

Fig. 2. Simulation framework

The structure of the simulation platform is shown in Fig. 2. VEINS provides a comprehensive set of simulation models of IEEE 802.11p and IEEE 1609.4 DSRC/WAVE network layers. Bidirectional coupling to a road traffic simulator is achieved by a node mobility model that interfaces with a running instance of a TraCI server such as SUMO. These models, together with the OMNeT++ simulation environment, provide the platform in which custom simulation models of protocols can be integrated. 


\section{VANETS AND AUTONOMOUS VEHICLES}

Autonomous vehicles (AV) are a promising driverless type of vehicle that can be part of the VANETs in the near future. AV need to communicate with others cars (V2V) and with the infrastructure around (V2I), in order to move in the roads interacting with the environment without problems, copying the human behaviour, adapting the driving according to the circumstances such as speed limit, pedestrian crossing the street or water in the road. Both kind of communications are necessary to detect pedestrians or obstacles (localization), movements of other cars (planning) to take a decision of what to do (execution).

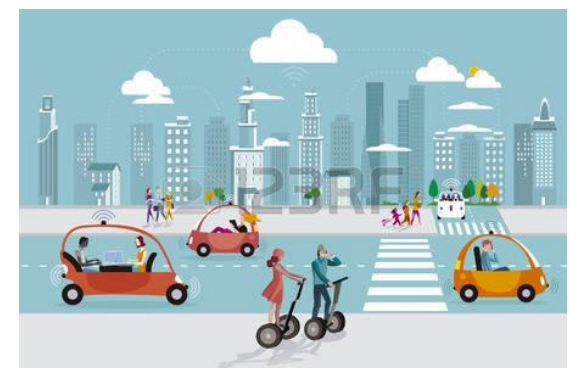

Fig. 3. Autonomous Driverless cars and people walking on the street. https://www.123rf.com

One major challenge most cities share is to find efficient ways to manage mobility. According to a study by Texas A\&M Transportation Institute in 2015 the time US commuters are stuck in traffic has risen by $133 \%$ since 1982 which equals a total of 42 hours. This means that on average drivers spend almost two full days in their vehicles per year. On average drivers loose an extra of 72 litres of fuel per year during traffic jams. In total, fuel emissions have gone up by $520 \%$ since the 1980 s, which is a huge strain on the environment.

On the other hand, as more and more people move to city outskirts, traffic congestion during rush hours is likely to become cities primer challenge. While most commuters drive into the city centre by car to get to work, most cities try to manage this problem by introducing traffic management systems and restrictive policies to regulate cars accessing the centre.

As traffic density increases, managing traffic and congestion will become more complex. In this context, AV and VANETs could alleviate drastically many issues related to mobility in cities, improving driving safety, decreasing pollution and reducing traffic congestion.

\section{VANETS, ELECTRICAL VEHICLE AND SMART ROAD LIGTING}

Finally, we are working on a global urban environment involving drivers, pedestrians and smart lighting of the roads. Looking for energy savings and reducing light pollution in the sky, we plan to link vehicle mobility behavior and road lighting. By modeling the mobility behavior of vehicles in the roads (either highways and city streets), road lighting could adapt to the actual lighting necessities throughout time, along the day and the week.

Besides, we also consider the presence of electrical vehicles (EVs) in the VANET. EVs have special needs, such as the reservation of an EVCS point during the required time to recharge their battery. An updated accurate knowledge of the mobility state in the city and the available EVCS points can help to manage better those reservations.

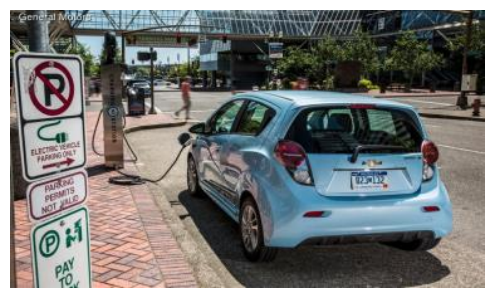

Fig. 4. Electrical vehicle charging station in a city. https://cdn.autocentre.ua/images/stories/2014/10/08/b/genera l-motors-planiruet-rasshirit-lineyku-eko-modeley-4.jpg

\section{CONCLUSIONS}

In this article, we have presented several research tasks that we are developing in our research team. The common topic is the design of algorithms, protocols and a realistic simulation platform to contribute in the development of vehicular adhoc networks (VANETs) in smart urban scenarios, focusing on video-streaming services with QoS provision.

\section{ACKNOWLEDGEMENTS}

This work was partly supported by the Spanish Government through the project TEC2014-54335-C41-R INcident monitoRing In Smart COmmunities, QoS and Privacy (INRISCO). Cristian Iza is recipient of a grant from Secretaria Nacional de Educación Superior, Ciencia y Tecnología SENESCYT. Ahmad Mohamad Mezher is a postdoctoral researcher with the Information Security Group (ISG) at the Universitat Politècnica de Catalunya (UPC).

\section{REFERENCES}

[1] Cristhian Iza, Ahmad M. Mezher, Mónica Aguilar, "An Adaptive Game-theoretical Distributed Dissemination Protocol for VANETs", Sensor Networks, In preparation.

[2] Omnet++, discrete event simulator. http://www.omnetpp.org

[3] Sumo - simulation of urban mobility. http://goo.gl/uvvD4N

[4] Inet framework. https://inet.omnetpp.org/

[5] Veins, vehicular network simulations. http://veins.car2x.org

[6] OpenStreetMap. http://www.openstreetmap.org

[7] Karp, B. and Kung, H.T. "GPSR: Greedy Perimeter Stateless Routing for wireless networks," MobiCom 2000

[8] Agusti Solanas, Úrsula Gonzalez-Nicohis, Antoni MartinezBallesté, "A Variable-MDAV-Based Partitioning Strategy to Continuous Multivariate Microaggregation with Genetic Algorithms", IJCNN 2010.

[9] Ahmad Mohamad Mezher and Mónica Aguilar Igartua, "Multimedia Multimetric Map-aware Routing protocol to send video-reporting messages over VANETs in smart cities", IEEE Transactions on Vehicular Technology, June 2017, DOI: $\underline{10.1109 / T V T .2017 .2715719}$ 